\title{
PROCESSING JUMP POINT OF LIDAR DETECTION DATA AND INVERSING THE AEROSOL EXTINCTION COEFFICIENT
}

\author{
Hailun Zhang ${ }^{1}$, Hu Zhao ${ }^{1,}$, Y Yapeng $\mathrm{Liu}^{2}$, Xingkai Wang ${ }^{2}$, Chang $\mathrm{Shu}^{2}$ \\ ${ }^{1}$ School of Electrical and Information Engineering, North MinZu University Yinchuan 750021, China - 809361497@qq.com, \\ zhaoh_1@yeah.net \\ ${ }^{2}$ School of computer science and engineering, North MinZu University Yinchuan 750021, China - 982843635@qq.com, \\ 1395585521@qq.com,410513982@qq.com
}

Commission III, WG III/8

KEY WORDS:Extinction Coefficient, Jump Point, Fitting, Interpolation, Invention Method

\begin{abstract}
:
For a long time, the research of the optical properties of atmospheric aerosols has aroused a wide concern in the field of atmospheric and environmental. Many scholars commonly use the Klett method to invert the lidar return signal of Mie scattering. However, there are always some negative values in the detection data of lidar, which have no actual meaning, and which are jump points. The jump points are also called wild value points and abnormal points. The jump points are refered to the detecting points that are significantly different from the surrounding detection points, and which are not consistent with the actual situation. As a result, when the far end point is selected as the boundary value, the inversion error is too large to successfully invert the extinction coefficient profile. These negative points are jump points, which must be removed in the inversion process. In order to solve the problem, a method of processing jump points of detection data of lidar and the inversion method of aerosol extinction coefficient is proposed in this paper. In this method, when there are few jump points, the linear interpolation method is used to process the jump points .When the number of continuous jump points is large, the function fitting method is used to process the jump points. The feasibility and reliability of this method are verified by using actual lidar data. The results show that the extinction coefficient profile can be successfully inverted when different remote boundary values are chosen. The extinction coefficient profile inverted by this method is more continuous and smoother. The effective detection range of lidar is greatly increased using this method. The extinction coefficient profile is more realistic. The extinction coefficient profile inverted by this method is more favorable to further analysis of the properties of atmospheric aerosol. Therefore, this method has great practical application and popularization value.
\end{abstract}

\section{INTRODUCTION}

At present, global climate changes attract people's great attention. Atmospheric aerosols is an important component of the earth-atmosphere system, which affects the balance of earth-atmosphere's radiation through direct radiation effects (scattering and absorption of solar radiation) and indirect radiation effects (mainly caused through changes in cloud characteristics) (Chen et al., 2011; Zhao et al., 2015; Cao et al., 2012). Different aerosol optical properties produce different atmospheric radiation effects. Therefore, the research of aerosol has always been an important topic for scientists. Lidar is an active remote sensing tool with high spatial and temporal resolution and continuous observation. Laser beam can interact with atmospheric aerosol particles, so the atmospheric optical parameters can be detected and inverted such as atmospheric extinction coefficient and backscatter coefficient (Ma et al., 2018).

The processing of lidar return signals has a crucial impact on the accurate inversion of optical parameters such as extinction coefficient. Many scholars have done a lot of research on processing of return signals and achieved remarkable results. Kovalev proposed an iterative algorithm that used the extinction coefficient of aerosol and atmospheric molecules to define a signal correction factor (Kovalev, 2003), and the return signals are corrected by an iterative algorithm to obtain the extinction coefficient of the aerosol. However, this method assumed that the boundary value of the aerosol extinction coefficient in a particular region is constant, so its accuracy cannot be guaranteed (Kovalev, 1993); He Yinghong used the least squares method to process the lidar return signals and estimate the boundary value of the extinction coefficient ( $\mathrm{He}$ et al., 2004). However,the essence of this method is based on the assumption that the atmosphere is uniform distribution. In fact, the atmospheric distribution is often not the case, so it is difficult to obtain accurate extinction coefficient boundary value.Susnik proposed a method for inversion of elastic lidar data (Susnik et al., 2014), which determines the boundary value of the extinction coefficient by minimizing the variance of the extinction coefficient in a defined region. However, the method works well for a single-component atmosphere. For the case, there may be two or more distributions, the spatial distribution of each aerosol must be determined and processed separately. The above studies are the determination of the boundary value in the process of lidar data inversion. However, if the selected boundary value is just a jump point, the extinction coefficient profile will have a large error or even cannot be inverted. The method proposed in this paper deals with the jump points in the detection data, so that the extinction coefficient profile can be inverted when any reasonable boundary value is selected.

"Corresponding author: Hu Zhao - zhaoh_1@yeah.net 
Sivakumar used wavelet technology to denoise the lidar signal and improve the signal-to-noise ratio of the lidar return signal (Sivakumar, 2017). However, this method has difficulty in determining the wavelet basis function.Ozcan et al. used the empirical mode decomposition method to filter the lidar data (Ozcan et al., 2015), but this method may filter out the effective signal at the same time, thus destroying the integrity of the effective signal.Cheng Zhi et al. proposed a hybrid method which combines Ensemble Empirical Mode Decomposition (EEMD) and singular value decomposition (SVD) to denoise the lidar signal and improve the signal-tonoise ratio of the original signal (Cheng et al., 2017). The above methods can suppress the noise of the lidar return signal to a certain extent, so as to improve the signal-to-noise ratio. But if there are jump points in the return signal, the detection range of lidar will greatly be reduced. In this paper, the detection range of lidar can be greatly improved by processing the jump point.

In the processing of lidar return signals, the denoising method and the determination of boundary values are studied by most scholars. At present, the jump point problem has been affecting the quality of lidar detection data. In this paper, the jump point and the invalid signal in the original return signal data of the lidar is directly processed. It is realized that the extinction coefficient profile can be successfully inverted when reasonable different far end boundary values are selected, which increases the effective detection range of lidar. It is more conducive to further process and analysis the lidar return signal.

\section{THE INVERSION METHOD OF EXTINCTION COEFFICIENT BY LIDAR}

The return data received by lidar is the energy value of aerosol and atmospheric backscattering. The lidar equation represents the relationship between the laser emission energy and the received energy. The optical parameters can be obtained by solving the lidar equation, such as the extinction coefficient of aerosol (Xiong et al., 2012). A lidar equation contains two unknowns, the atmospheric extinction coefficient $\sigma(r)$ and the backscatter coefficient $\beta(r)$. Many methods have been proposed to deal with this problem, such as Collis slope method、Klett method and Fernald method are extensive used (Collis and Russell, 1976; Klett, 1981; Fernald, 1984). The Klett method overcomes the limitation of uniform atmosphere and has been used widely.

Assume $\beta(r)$ and $\sigma(r)$ have the following nonlinear relationship:

$$
\beta(r)=c_{1} \sigma^{k}(r)
$$

where $\mathrm{c}_{1}$ is a constant, it has nothing to do with the inversion result. The value of $\mathrm{k}$ is related to the lidar wavelength and the properties of aerosols. The value range of $\mathrm{k}$ is $0.67-1$, and $\mathrm{k}=1$ is used in this paper. Assume that $r \leq r_{m}, \mathrm{r}_{\mathrm{m}}$ is the boundary value of the range, generally the maximum effective detection range is selected, then the extinction coefficient at $r$ can be expressed as:

$$
\sigma(r)=\frac{e^{\{[S(r)-S(r) / k]\}}}{\left\{\sigma^{-1}\left(r_{m}\right)+\frac{2}{k} \int_{r}^{r_{m}} e^{\left\{\left[S(r)-S\left(r_{m}\right) / k\right]\right\}} d r\right\}}(2)
$$

Where $S(r)=\ln \left[r^{2} P(r)\right]$, is the logarithmic form of the range squared correction signal. It can be seen from equation (2) that both the numerator and the denominator contain $\mathrm{S}(\mathrm{r})$, and the true value of the logarithmic function must be greater than zero. when the extinction coefficient is inverted using the unprocessed data, the selected far end boundary value is exactly the jump point, it make the logarithmic function to be meaningless, and the calculation results will be wrong, so that the extinction coefficient profile cannot be successfully inverted. When the effective signal point is selected as the boundary value, there are still some jump points in the unprocessed return data, which will lead to the calculation error of the denominator in the formula at the jump points. Although the extinction coefficient profile can be successfully inverted, but the extinction coefficient profile is discontinuous at the jump points, and the error is large.

\section{PROCESSING JUMP POINT AND NUMERICAL SIMULATION}

\subsection{Processing Method For Jump Points}

When inverting the extinction coefficient with the lidar equation, it is often encountered that some negative values in the detection data of lidar, which have no actual meaning, and these negative points are jump points. As a result, when the far end point is selected as the boundary value, the inversion error is too large to successfully invert the extinction coefficient profile.

In order to solve the problem, a method of processing jump point of detection data of lidar and the inversion method of aerosol extinction coefficient is proposed in this paper. In this method, when there are few jump points, the linear interpolation method is used to process the jump points. When the number of continuous jump points are large, the function fitting method is used to process the jump points. 


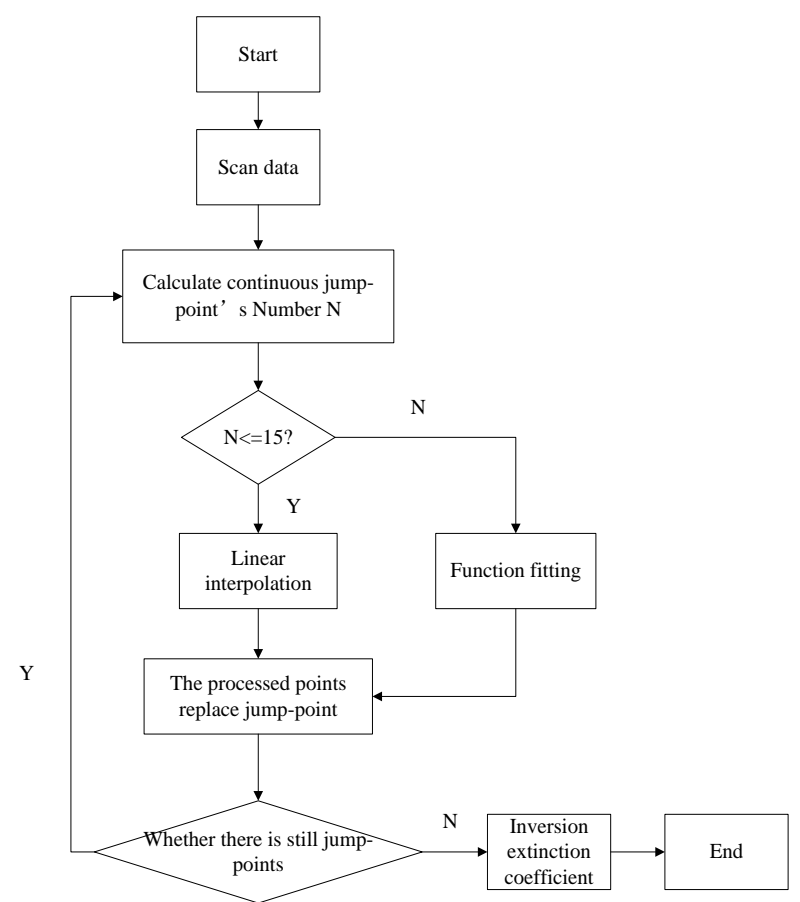

Figure 1. Flow chart of the jump points processing algorithm for the detection data

Figure 1 is a flow chart of the jump points processing algorithm for detection data. First, the original data is scanned. When the number of consecutive jump points $\mathrm{N}$ is small, in this paper we take $\mathrm{N}$ as 15 , it can be think that the atmosphere is homogeneous at this range, so the jump points are processed by linear interpolation. When the consecutive jump points are processed, for the more consecutive jump points received, they are processed by function fitting. When performing function fitting, we try to select the fitting function whose values are basically on the positive half of the y-axis and have a strong coincidence with the effective signal points. It is enable most of the jump points to be corrected and match the trend of effective signal points better. When the function-fitting data still have negative points, this process continues until all the jump points are successfully corrected. Finally, the correction data obtained by linear interpolation and function fitting are used to replace the jump points of the original data, and the extinction coefficient is calculated by klett method.

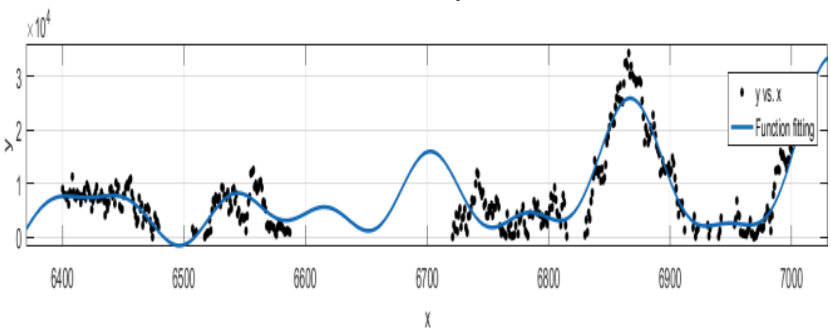

Figure 2. Schematic diagram of function fitting of data in the interval points of $6400-7000$

Figure 2 shows the schematic diagram of the function fitting of the signal points in the interval of 6400-7000. In this interval, the interval containing a small number of jump points has been corrected by linear interpolation, and there are more continuous jump points in the intervals of 6480-6518 $65588-6720$ and 6815-6830. According to the known effective signal points, function fitting is applied to the jump points, and the data containing jump points are corrected to effective data that can be used. In this paper, MATLAB fitting toolbox is used to select the fitting function. The selected fitting function is 'sum of $\sin$ '. It has eight types. The basic type is $\mathrm{f}(\mathrm{x})=\mathrm{a}_{1} * \sin \left(\mathrm{b}_{1} * \mathrm{x}+\right.$ $\mathrm{c}_{1}$ ), the fitted function curve is in line with the trend of lidar echo data at the far end. Within this interval, the fitting function selected in this paper is the sixth type, whose form is $f(x)=a_{1} * \sin \left(b_{1} * x+c_{1}\right)+a_{2} * \sin \left(b_{2} * x+c_{2}\right)+a_{3} * \sin \left(b_{3} * x+c_{3}\right)+$ $\mathrm{a}_{4} * \sin \left(\mathrm{b}_{4} * \mathrm{x}+\mathrm{c}_{4}\right)+\mathrm{a}_{5} * \sin \left(\mathrm{b}_{5} * \mathrm{x}+\mathrm{c}_{5}\right)+\mathrm{a}_{6} * \sin \left(\mathrm{b}_{6} * \mathrm{x}+\mathrm{c}_{6}\right)$.

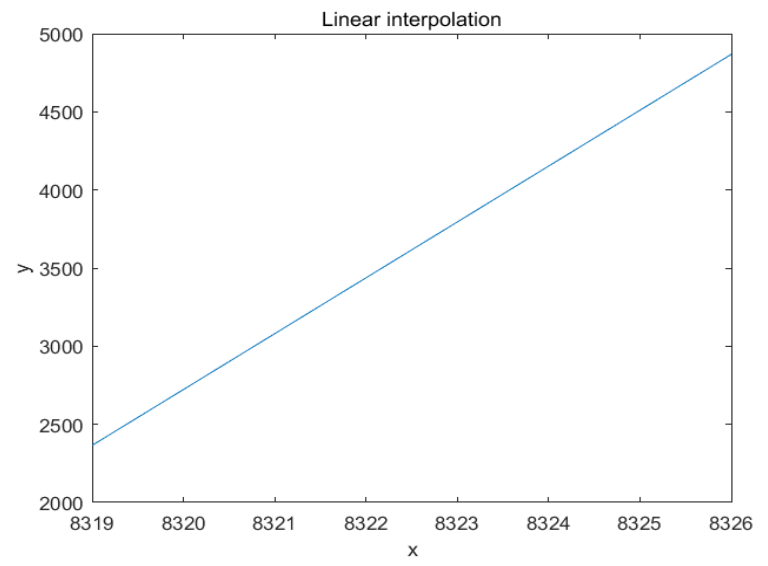

Figure 3. Schematic diagram of linear interpolation of 89839294 interval data

Figure 3 shows the linear interpolation diagram of the 89839294 interval points, in which 8983 and 9294 are known effective signal points, and 7 points in the 9294-9293 interval are jump points. Due to the small number of jump points, it can be considered that the atmospheric aerosol distribution is uniform in this interval, so the jump points could be processed by linear interpolation.

\subsection{Numerical Simulation}

When using the klett inversion method to invert the lidar return signal, the ratio of atmospheric molecular extinction coefficient to backscatter coefficient is $8 / 3 \pi$, and the ratio of aerosol extinction coefficient to backscatter coefficient (lidar ratio) is 50. The extinction coefficient and backscatter coefficient values for atmospheric molecules can be obtained by the US standard atmospheric model (Sun et al., 1986; Yan et al., 2001). In this paper, the measured $1064 \mathrm{~nm}$ lidar return signal is used for numerical simulation. After the actual measured echo data is processed by the method of this paper, then the klett method is used to calculate the extinction coefficient. 


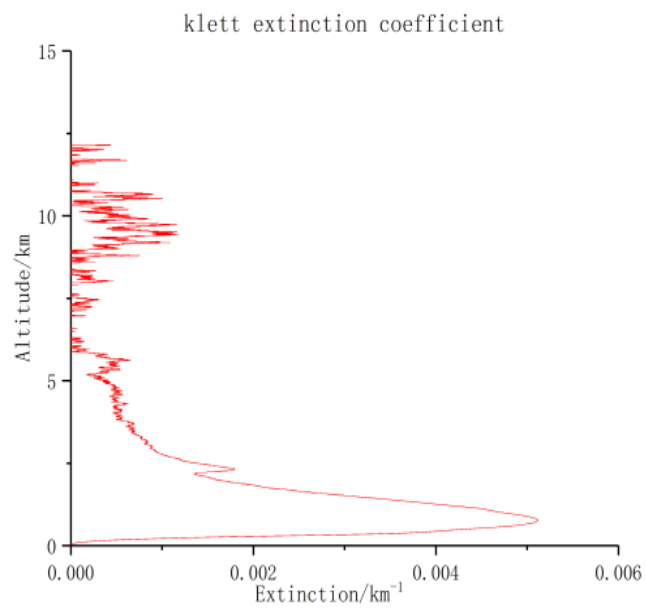

Figure 4. Extinction coefficient profile with jump points data inversion when the 9100th detection point is selected as the boundary point.

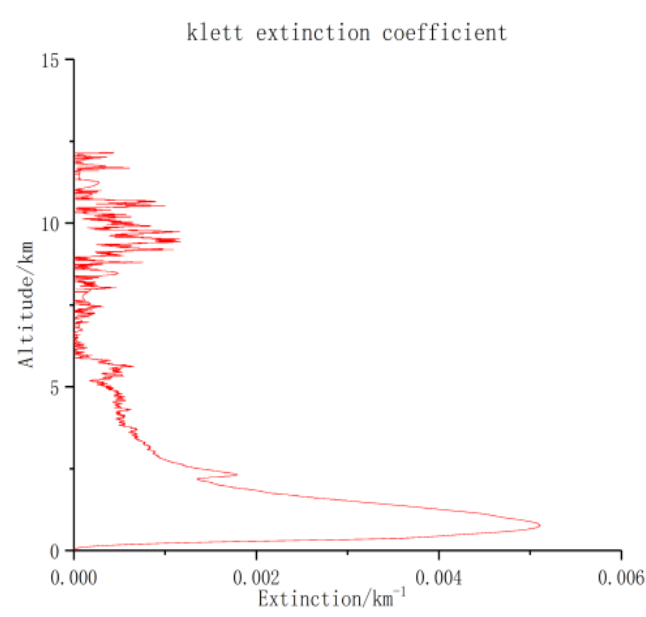

Figure 5. Extinction coefficient profile without jump points data inversion when the 9100th detection point is selected as the boundary point

In the case, the same boundary condition is selected, Figure 4 shows the extinction coefficient profile inverted by the data with the jump point. Figure 5 shows the extinction coefficient profile inverted by the data without the jump point. It can be clearly seen that in Fig. 4 within the range of $6.3-6.9 \mathrm{~km}, 7.6-$ $7.9 \mathrm{~km}$ and $11.1-11.5 \mathrm{~km}$, the extinction coefficient profile has obvious discontinuities, and the extinction coefficient profiles has not been successfully inverted in these ranges. However, the extinction coefficient profile obtained in Fig. 5 is successfully inverted in these ranges, and the extinction coefficient profile obtained by the inversion is more continuous and smoother, which not only greatly increases the effective detection range of the lidar, but also makes the extinction coefficient profile is more in line with the actual situation and is more conducive to further analysis of the subsequent atmospheric aerosol extinction coefficient profile.

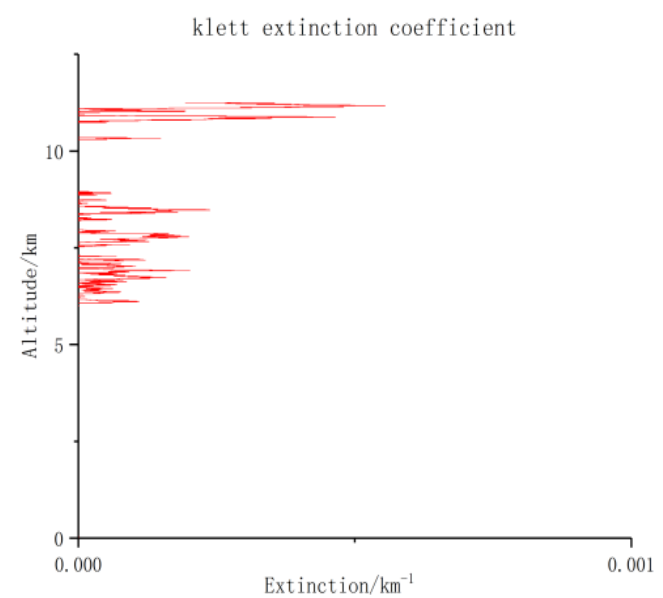

Figure 6 . The extinction coefficient profile with jump points data inversion when the 8500th detection point is selected as the boundary point

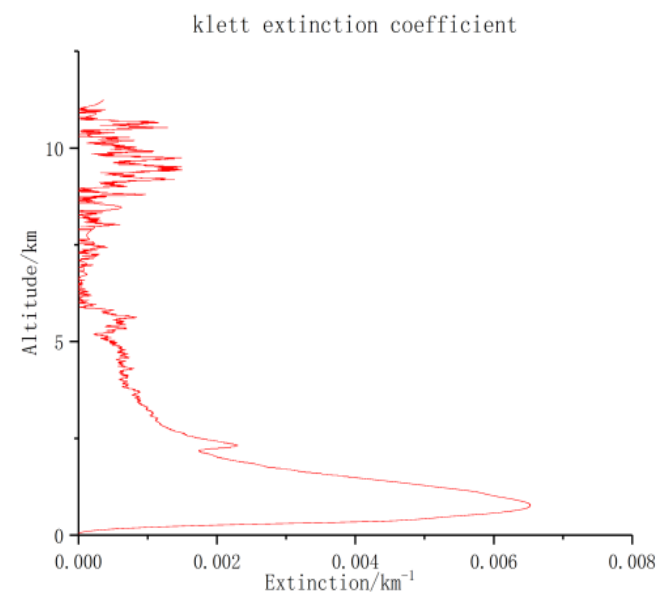

Figure 7. The extinction coefficient profile without jump points data inversion when the 8500th detection point is selected as the boundary point

In the case, the same boundary condition is selected. Figure 6 shows the extinction coefficient profile obtained by the detection data with the jump point, which has not successfully inverted the extinction coefficient profile. Figure 7 shows the extinction coefficient profile obtained by the detection data without jump points, which not only inverted the extinction coefficient profile successfully, but also makes the extinction coefficient profile more continuous and smoother. After a large number of numerical simulations, it can be known that when the extinction coefficient is inverted by the method in this paper, the extinction coefficient profile can be successfully inverted by selecting different far end boundary values arbitrarily. Therefore, this method is expected to be applied to the calculation of aerosol extinction coefficient profiles in lidar remote sensing detection in the future. 


\begin{tabular}{|c|c|c|c|}
\hline $\begin{array}{c}\text { Boundary } \\
\text { value }\end{array}$ & $\begin{array}{c}\text { Yes/No invert the extinction } \\
\text { coefficient profile with jump- } \\
\text { point }\end{array}$ & $\begin{array}{c}\text { Yes/lo invert the extinction } \\
\text { coefficient profile without jump- } \\
\text { point }\end{array}$ & Relative error \\
\hline 8000 & Yes & Yes & 0.0032 \\
\hline 8100 & Yes & Yes & 0.003 \\
\hline 8200 & No & Yes & 1.3306 \\
\hline 8300 & Yes & Yes & 0.0063 \\
\hline 8400 & No & Yes & 2.0903 \\
\hline 8500 & No & Yes & 1. 3939 \\
\hline 8600 & No & Yes & 1. 1049 \\
\hline 8700 & No & Yes & 1.3813 \\
\hline 8800 & Yes & Yes & 0.0064 \\
\hline 8900 & No & Yes & 1.6972 \\
\hline
\end{tabular}

Table 1. The errors of extinction coefficient profiles under different boundary conditions

Table 1 shows 10 points are selected as the boundary value starting from the 8000th detection point, and the selected adjacent two boundary values are separated by 100 signal points. The extinction coefficient profiles are inverted and their relative error values are calculated in the case where the detection data with jump points and without jump points. According to numerical simulation, it can be known that removing jump points can invert the extinction coefficient profile by selecting any reasonable boundary value. However, the detection data with the jump points are among the 10 randomly selected boundary values. Only four extinction coefficient profiles can be successfully inverted, six extinction coefficient profile cannot be inverted. When the effective data point is selected as the boundary value, both of them can inverse the extinction coefficient profile, and the relative error is small; When the jump points are selected as the boundary values, their relative errors is very large. Therefore, the jump points have a crucial influence on the inversion of the lidar data. If there are jump points in the detected data, the result of the inversion will be seriously affected. However, after the detection data is processed by the method of this paper, the problem will be overcomed.

\section{CONCLUSION}

In this paper, the method of processing jump point of lidar detection data and the inversion method of aerosol extinction coefficient is proposed. In this method, when there are few jump points, it can be considered that the atmospheric distribution is uniform within this distance, and the linear interpolation method is used to process the jump points. When the number of continuous jump points is large, the function fitting method is used to process the jump points. The feasibility and reliability of this method are verified by using actual lidar data. The results show that the extinction coefficient profile can be successfully inverted when different remote boundary values are chosen. The extinction coefficient profile inverted by this method is more continuous and smoother. The effective detection range of lidar is greatly increased using this method.The extinction coefficient profile is more realistic. The extinction coefficient profile inverted by this method is more favorable to further analysis of the properties of atmospheric aerosol. Therefore, this method has great practical application and promotion value, and it is expected to be applied to the calculation of aerosol extinction coefficient profiles in lidar remote sensing detection in the future.

\section{ACKNOWLEDGEMENTS}

This research was funded by the National Natural Science Foundation of China (Grant No. 61865001) and the Ningxia Natural Science Foundation (Grant No. 2018AAC03103).

\section{REFERENCES}

Chen, S., Zhang Y., Chen, H., Guo, P., Wang, Y., 2011. Comparing methods for retrieving aerosol extinction coefficient with U.S. Standard Atmospheric Model and Temperature Gradient. International Conference on Remote Sensing, IEEE, 2043-2046. 10.1109/RSETE.2011.5964706.

Cao, N., Shi, J., Yang, F., Yan, P., 2012. Discussion of the relationship between the aerosol extinction coefficient error and background noise. Applied Physics B, 108(4), 945950.10.1007/s00340-012-5204-5.

Cheng, z., He, F., Jing, X., et al., 2017. Denoising of Lidar Signal Based on Ensemble Empirical Mode Decomposition and Singular Value Decomposition. Photonics Journal, 46(12). 1201003.

Collis, R. T. H., Russell, P. B., 1976. Lidar measurement of particles and gases by elastic backscattering and differential absorption. 71-151. 10.1007/3-540-07743-X_18.

Fernald, F. G., 1984. Analysis of atmospheric lidar observations: some comments. Applied Optics, 23(5), 652. 10.1364/AO.23.000652.

He, Y., Zheng, Y., Cheng, J., et al., 2004. Estimation of extinction efficient boundary value with least-square fitting for lidar return signal. Chinese Journal of Quantum Electronics, 21(6), 879-883. 10.1016/j.csr.2003.12.006.

Kovalev, V. A., 2003. Stable near-end solution of the lidar equation for clear atmospheres. Applied Optics, 42(3), 585-591. 10.1364/AO.42.000585.Kovalev V. A., 1993. Lidar measurement of the vertical aerosol extinction profiles with range-dependent backscatter-to-extinction ratios. Appl Opt, 32(30), 6053-6065.

Klett, J. D., 1981. Stable analytical inversion solution for processing lidar returns. Applied Optics, 20(2), 211-20. 10.1364/AO.20.000211.

Ma, Y., Liu, J., Chen, N., Gao, H., Xiong, X., 2018. Determination of the boundary value of the aerosol extinction coefficient and its effects on the extinction coefficient profile of aerosol in lower atmosphere. Optik, 160, 283-297. 10.1016/j.ijleo.2018.01.116.

Ozcan, A. H., Unsalan, C., 2015. LiDAR height data filtering using Empirical Mode Decomposition. Signal Processing and Communications Applications Conference, SIU 2015Proceedings, IEEE. 10.1109/SIU.2015.7130058.

Susnik, A., Holder, H., Eichinger, W., 2014. A minimum variance method for lidar signal inversion. Journal of Atmospheric and Oceanic Technology, 31(2), 468-473. 10.1175/jtech-d-13-00125.1.

Sivakumar, V., 2007. De-noising LiDAR signal using wavelet technique. Lidar Remote Sensing for Environmental 
Monitoring VIII. International Society for Optics and Photonics. 6681. 10.1117/12.739450.

Sun, J., et al., 1986. Laser Atmospheric Detection. Science Press. 48-81.

Xiong, X., Jiang, L., Feng, S., 2012. Return signals processing method of Mie scattering lidar. Infrared and Laser Engineering, 41(1), 89-95.
Yan, J., Gong, S., Liu, Z., 2001. Environmental Monitoring Lidar. Science Press. 67-78.

Zhao, H., Hua, D., Di, H., Hou, X., Mao, J., 2015. Development of all time multi-wavelength lidar system and analysis of its signal to noise ratio. Chinese Journal of Lasers, 42(1), 0113001. 10.3788/CJL201542.0113001 